\title{
Coagulation-dependent mechanisms and asthma
}

\author{
Michael A. Matthay and John A. Clements \\ Cardiovascular Research Institute, University of California, San Francisco, San Francisco, California, USA.
}

\begin{abstract}
In several clinical disorders, there are interactions between inflammation-dependent tissue injury and thrombin formation, fibrin deposition, and impaired fibrinolysis. New evidence generated from a mouse model of allergic airway hyperreactivity suggests that disordered coagulation and fibrinolysis may contribute to the pathogenesis of asthma (see the related article beginning on page 104). The inflammatory mechanisms that lead to airway smooth muscle contraction and airway hyperresponsiveness may be associated with accumulation of extravascular fibrin, plasma exudates, and inflammatory cells that can lead to airway closure.
\end{abstract}

In patients with severe sepsis, abnormalities in coagulation play a central role in the pathogenesis of multiple organ system failure, including acute lung injury $(1,2)$. Procoagulant activity and impaired fibrinolysis are important early findings in the distal airspaces of patients with lung injury, predisposing to fibrin deposition and alveolar epithelial injury $(3,4)$. Disorders of fibrin turnover have also been implicated in experimental models of lung fibrosis (4) and recently in the regulation of the inflammatory response in neuroinflammatory diseases such as multiple sclerosis (5). In this issue of the JCI, Wagers et al. (6) propose the novel hypothesis that fibrin deposition and abnormalities in the coagulation and fibrinolytic pathways in the distal airways of the lung significantly contribute to airway hyperresponsiveness and airway closure in asthma.

The authors discovered fibrin deposition on the luminal surface of the airway epithelium in a patient who died in status asthmaticus. Then, using a standard mouse model of allergic airway hyperresponsiveness, they documented fibrin in the distal airways and the alveoli; greater quantities of D-dimer (a breakdown product of cross-linked fibrin) in the bronchoalveolar lavage; and an increase in plasminogen activator inhibitor type-1 (PAI-1) activity in the lung homogenates (6). The latter would be expected to promote accumulation of fibrin by suppressing fibrinolysis. Administrations

Nonstandard abbreviations used: plasminogen activator inhibitor type-1 (PAI-1).

Conflict of interest: The authors have declared that no conflict of interest exists.

Citation for this article: J. Clin. Invest. 114:20-23 (2004). doi:10.1172/JCI200422288. of a nebulized fibrinolytic agent (tissue plasminogen activator) or anticoagulation with a thrombin inhibitor (hirudin) reduced airway hyperresponsiveness in the sensitized mice. To test the contributions of thrombin and fibrinogen to airway hyperresponsiveness, these molecules were nebulized and administered to mice separately and in combination. Only the thrombin-fibrinogen combination induced all of the changes in respiratory resistance and lung elastance that occurred in the allergic, airway-hyperresponsive mice. These results suggest there is a potentially important connection between coagulation events and asthmalike responses.

To explain their findings, the authors propose that the presence of fibrin in the distal airways neutralizes surfactant, resulting in an increase in surface tension that would favor collapse of distal airways (6). The rightward shift and the decreased hysteresis of the pressure volume curve from the mice with allergic airway inflammation are interpreted as indicating an increase in distal airway closure resulting from a decrease in functional surfactant in these airways. Further, they believe that the reversal of these findings with aerosolized tissue plasminogen activator is consistent with a decrease in distal airway surface tension that reflects enhanced function of surfactant.

\section{Surface tension and airway closure}

Is it likely that inhibition of surfactant at the terminal and respiratory bronchial level could significantly contribute to airway closure? Reduced surfactant function has been reported in sputum and bronchoalveolar lavage from patients with asthma (7). Plasma extravasation into the distal airways, an important feature of asthma, would supply plasma proteins that could inactivate surfactant (8). Also, surfactant itself could have a procoagulant effect that can contribute to fibrin formation in the distal airspaces of the lung (9). However, there are difficulties in explaining distal airway closure primarily on the basis of impaired surfactant function. First, based on the available pool of surfactant in the lung, the area of surface film in the alveoli is several thousand times that in the bronchioles, and this reservoir of surface active material lies less than $2 \mathrm{~mm}$ from the distal airways (Figure 1). Surfactant films can move rapidly in wet tubes the size of terminal bronchioles (10). For these reasons it seems likely that surface tension is similar in bronchioles and alveoli, although no direct in situ measurements in undisturbed distal airways are available. Also, with the large reservoir of surfactant nearby, the quantity of fibrin needed to inhibit surfactant would be very high. One study has shown that fibrin monomer inhibited surfactant function in a ratio of $1: 3$ by weight in vitro (11). If this ratio were relevant to in vivo conditions, the quantity of fibrin harvested by lavage in the study of Wagers et al. (6) would be too small by a factor of more than 28,000 to account for surfactant inhibition. It would be too small by a factor of about 10 if all of the fibrin were concentrated in the distal airways. Both Figure 1 and ref. 12 indicate that much of the fibrin is deposited in the alveoli. Because of tethering of distal airways to lung parenchyma, an increase in alveolar surface tension would tend to dilate rather than collapse them (13).

\section{Airway secretions, edema, and smooth muscle tone}

Are there alternative explanations for the interesting findings in this study? Based on a simple experimental model, if the length of a capillary tube is three or more times its diameter - a shape that approximately corresponds to that of human bronchioles - then there is a critical volume of luminal liquid that will cause the fluid to contract and obstruct the lumen (14). The critical 


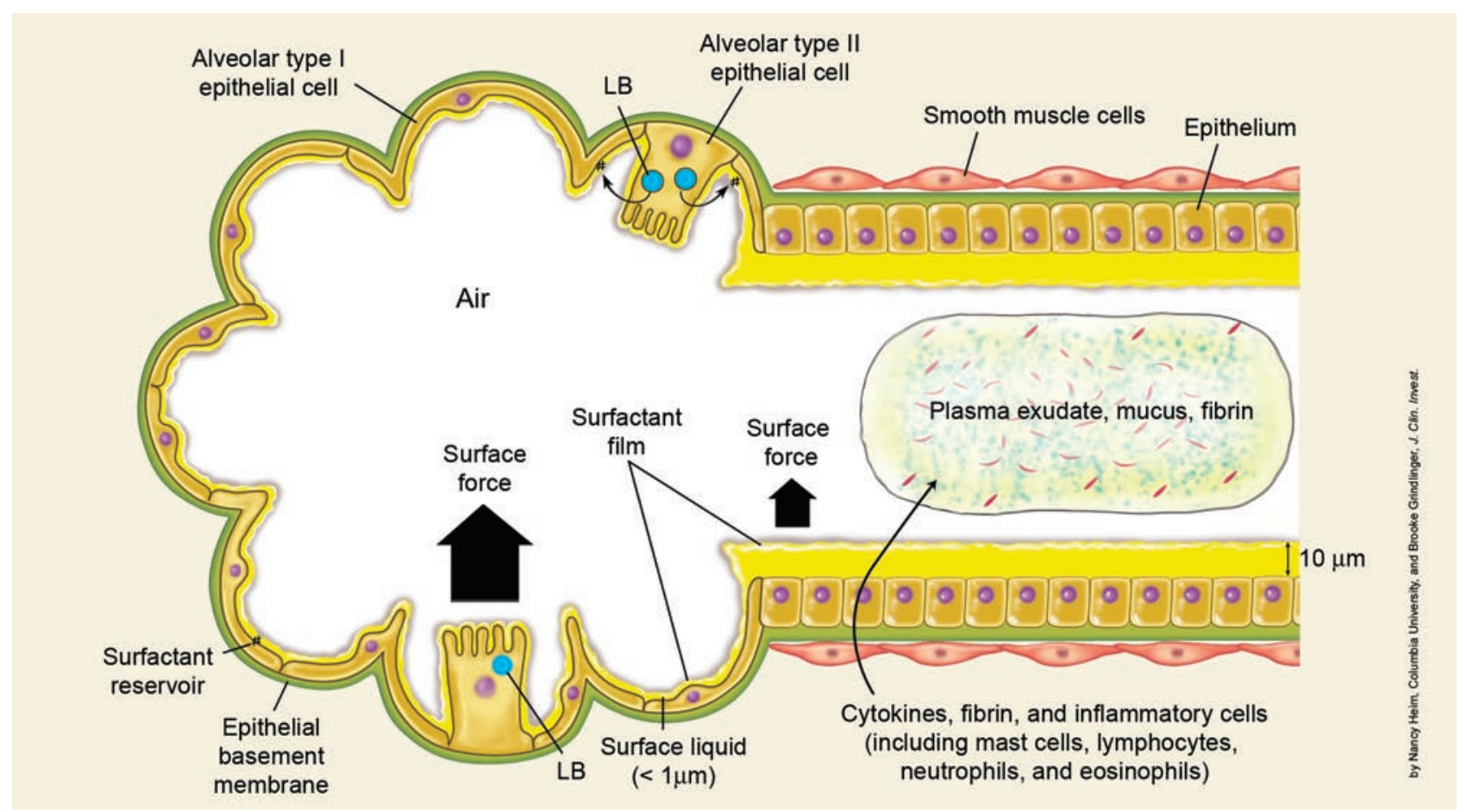

\section{Figure 1}

Schematic diagram of the alveoli and a terminal airway. Most of the alveolar surface is covered by thin type I epithelial cells. The type II epithelial cells secrete surfactant from lamellar bodies (LBs) into the thin layer of alveolar surface liquid lining the alveoli. The terminal airways are lined by cuboidal epithelial cells and have bands of smooth muscle cells in their walls. A thin liquid layer, the surface tension of which is decreased by the presence of surfactant, lines both alveoli and terminal airways. Because the fluid layer is continuous in the alveoli and the small airways, surfactant can move readily between them, tending to equalize surface tension. The component of pressure due to surface tension is expected to be about four times as large in alveoli as in terminal airways because the radii of curvature are smaller in the quasi-spherical alveoli compared with those in the quasi-cylindrical airways. Thus, the tendency to collapse from surface tension is greater in the alveoli than in the terminal airways. In addition, alveolar surface tension can make a strong contribution to the tethering forces that tend to expand the terminal airways. The presence of plasma from cytokine-dependent inflammation can collect in the distal airways. When inflammation associated with asthma occurs, plasma exudate, mucus, and fibrin accumulate in the airways, potentially leading to airway closure (see Figure 2). The cellular and molecular basis for airway inflammation in asthma was reviewed recently (17).

ratio is $V_{C} / D^{3}=0.7$, where $V_{C}$ is the critical volume and $D$ is the internal diameter of the tube. The mechanisms that would enhance this kind of airway closure would be exudation of plasma into the airway lumen, as well as airway wall edema, and the active contraction of smooth muscle (Figure 2). In the mouse studies by Wagers et al. (6), fibrinolytic therapy with tissue plasminogen activator prevented hyperresponsiveness and increased D-dimer release, indicating that fibrinolysis had occurred. However, this therapy could have also reduced the intensity of the inflammatory response, an effect that would reduce the volume of extravasated plasma into the airway lumen and the intensity of smooth muscle contraction. A reduction in extravascular plasma and smooth muscle contraction would improve the hysteresis of the pressure volume curve and attenuate the rises in respiratory resistance and lung elastance. The administration of thrombin and fibrinogen that reproduced the severity of lung function abnormalities and fibrin deposition may also be associated with inflammatory responses that enhance smooth muscle contraction, effects that may also be magnified by released fibrinopeptides (15). There is an abundant literature that links thrombin generation and impaired fibrinolysis to inflammation and tissue injury $(2,16)$. Interestingly, a recent review in the JCI (17) emphasized that the events downstream from release of IL-13, an important cytokine in Th2 inflammatory responses in asthma, may involve TGF- $\beta$, a molecule that can be activated by a plasmin-dependent pathway. TGF- $\beta$ could be important because it may play a role in the subepithelial airway fibrosis in asthma and it can upregulate gene expression of PAI-1 (17), one of the markers of impaired fibrinolysis that Wagers et al. (6) found in the lungs of the asthmatic mice.
Other investigators have recently reported that administration of activated protein $C$, a naturally occurring endogenous anticoagulant that also enhances fibrinolysis, reduced obstruction (as inferred from indirect plethysmography) in a standard mouse model of allergic asthma, primarily by inhibiting immunologic and inflammatory responses induced by Th2 cytokines (18). Also, the proinflammatory environment of inflamed airways could contribute to enhanced mucus secretion from goblet cells in more proximal airways, and the plasma exudation and deposition of fibrin may result in a feedback process that enhances accumulation of liquid and mucus in the airways (19). Plasma exudation itself may be amplified by several mechanisms, including the release of VEGF, a molecule that has been identified in asthmatic airways and is well known for 


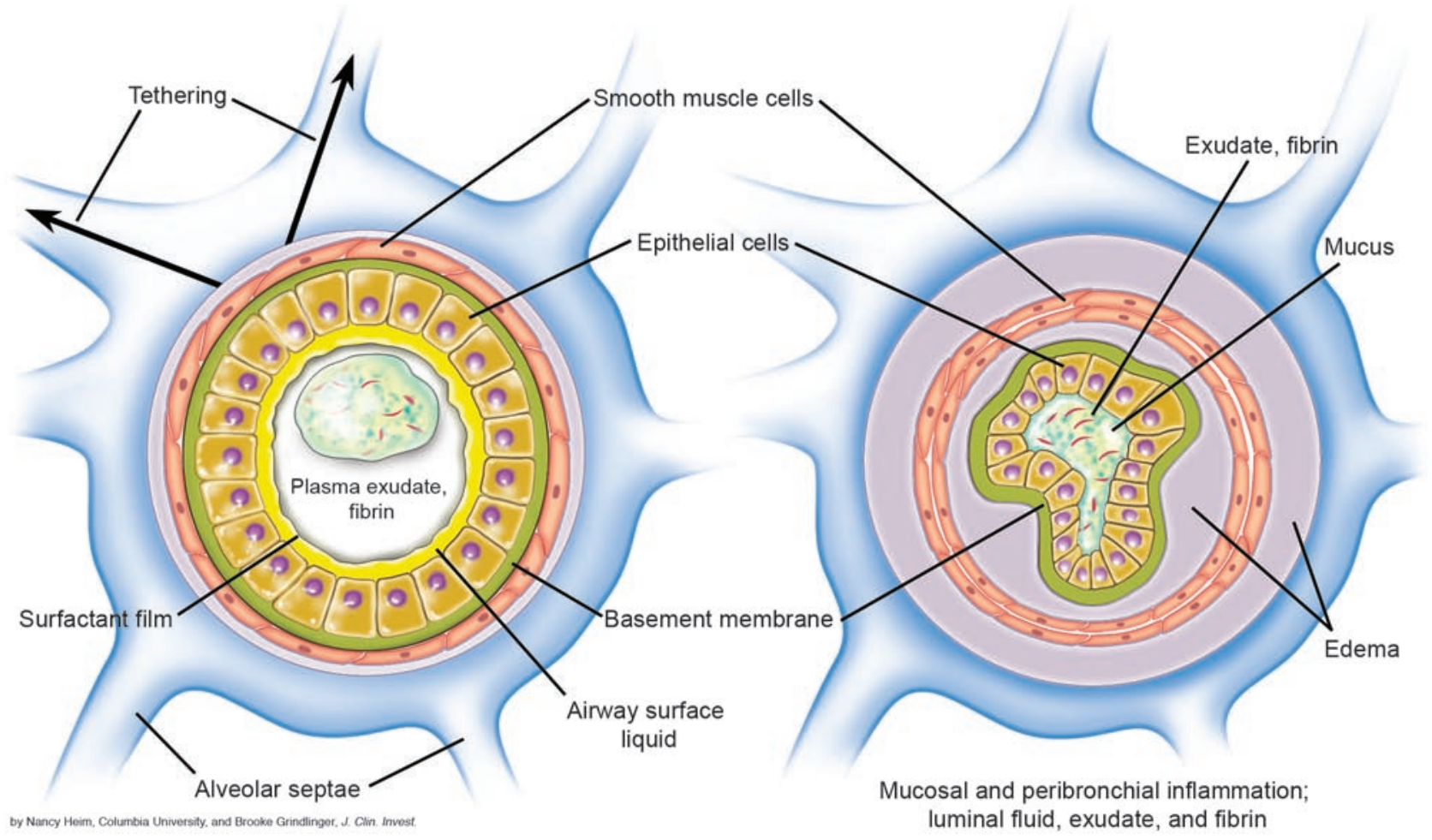

Figure 2

Schematic cross-sectional diagram of terminal airways illustrating the transition from an opened to a closed airway. Contraction of smooth muscle cells favors airway constriction coupled with variable quantities of plasma proteins in the airway wall and the airway lumen, including fibrin deposition (see Figure 1 in Wagers et al. [ref. 6]). Normally there are tethering forces that will tend to expand terminal airways, while the quantity and quality of airway liquid (and mucus) favor airway obstruction. Although surface tension in the distal airways would be expected to play a minor role in decreasing patency under these conditions, administration of exogenous surfactant might help expel occluding material by dilution, dispersion, and rapid lowering of surface tension at the surfaces of the plugs.

its ability to increase vascular permeability (20). In the presence of plasma exudation, airway closure could be amplified by both airway wall edema and accumulation of liquid and mucus in the airspaces (Figures 1 and 2). If some airways remained closed throughout the respiratory cycle, blocking access of alveolar surfactant, clearance of surfactant above the plugs into central airways would be expected to raise surface tension and impede removal of intraluminal material. The reported benefit of aerosolized surfactant in one study of human asthma (21) might be explained by enhanced expulsion of plugs from distal airways during expiration.

In conclusion, the observations in this study provide evidence that fibrin accumulation and abnormalities in procoagulant and fibrinolytic pathways are associated with airway hyperresponsiveness in experimental asthma (6). The mechanisms that account for these findings are probably not explained simply by inactivation of surfactant in the distal airways.
Nevertheless, there are several other tenable explanations, and further study is now needed to identify the contribution of specific arms of the coagulation pathways. Because elevated PAI-1 levels are linked to excess fibrin formation and perhaps airway remodeling, as well as possibly a propensity to develop human asthma based on PAI-1 polymorphisms (22), it would be interesting to know whether PAI-1-knockout mice are protected in mouse models of asthma. In the context of human asthma, it is known that there is activation of the coagulation system and that thrombin is increased in the airways, a finding that is associated with airway inflammation (23). More work should be directed toward establishing whether abnormalities in the coagulation and fibrinolytic system are truly associated with mild, moderate, or severe asthma. Perhaps some of the newly available anticoagulants and fibrinolytic agents could be tested for their efficacy in the prevention or treatment of human asthma.

\section{Acknowledgments}

This commentary was supported in part by NIH grants HL51854, HL51856, P50HL74005, and HL24075.

Address correspondence to: Michael A. Matthay, Cardiovascular Research Institute, University of California, HSW-825, 505 Parnassus Avenue, San Francisco, California 94143-0130, USA. Phone: (415) 353-1206; Fax: (415) 353-1990; E-mail: mmatt@itsa.ucsf.edu.

1. Bernard, G.R., et al. 2001. Efficacy and safety of recombinant activated protein $\mathrm{C}$ for severe sepsis. N. Engl. J. Med. 344:699-709.

2. Esmon, C.T. 2002. Protein C pathway in sepsis. Ann. Med. 34:598-605.

3. Ware, L.B., Fang, X., and Matthay, M.A. 2003. Protein $\mathrm{C}$ and thrombomodulin in acute lung injury. Am. J. Physiol. Lung Cell Mol. Physiol. 285:L514-L521.

4. Idell, S. 2002. Endothelium and disordered fibrin turnover in the injured lung: newly recognized pathways. Crit. Care Med. 30(5 Suppl.):S274-S280.

5. Akassoglou, K., et al. 2004. Fibrin depletion decreases inflammation and delays the onset of demyelination in a tumor necrosis factor transgenic mouse model for multiple sclerosis. Proc. 
Natl. Acad. Sci. U. S. A. 101:6698-6703.

6. Wagers, S.S., et al. 2004. Extravascular fibrin, plasminogen activator, plasminogen activator inhibitors, and airway hyperresponsiveness. J. Clin. Invest. 114:104-111. doi:10.1172/JCI200419569.

7. Jarjour, N.N., and Enhorning, G. 1999. Antigeninduced airway inflammation in atopic subjects generates dysfunction of pulmonary surfactant. Am. J. Respir. Crit. Care Med. 160:336-341.

8. Yager, D., Kamm, R.D., and Drazen, J.M. 1995 Airway wall liquid: sources and role as an amplifier of bronchoconstriction. Chest. 107(3 Suppl.):105S-110S.

9. Taylor, F.B., Jr., and Abrams, M.E. 1966. Effect of surface active lipoprotein on clotting and fibrinolysis, and of fibrinogen on surface tension of surface active lipoprotein. Am. J. Med. 40:346-350.

10. Putz, G., Goerke, J., Taeusch, H.W., and Clements, J.A. 1994. Comparison of captive and pulsating bubble surfactometers with the use of lung surfactants. J. Appl. Physiol. 76:1425-1431.

11. Seeger, W., Stohr, G., Wolf, H.R., and Neuhof, H. 1985. Alteration of surfactant function due to protein leakage: special interaction with fibrin monomer. J. Appl. Physiol. 58:326-338.

12. Schmiedl, A., et al. 2003. Increase of inactive intraalveolar surfactant subtypes in lungs of asthmatic brown Norway rats. Virchows Arch. 442:56-65.

13. Mead, J., Takishima, T., and Leith, D. 1970. Stress distribution in lungs: a model of pulmonary elasticity. J. Appl. Physiol. 28:596-608.

14. Kamm, R.D., and Schroter, R.C. 1989. Is airway closure caused by a liquid film instability? Respir. Physiol. 75:141-156.

15. Bayley, T., Clements, J.A., and Osbahr, A.J. 1967. Pulmonary and circulatory effects of fibrinopeptides. Circ. Res. 21:469-485.

16. Coughlin, S.R. 2000. Thrombin signaling and protease-activated receptors. Nature. 407:258-264.

17. Elias, J.A., et al. 2003. New insights into the pathogenesis of asthma. J. Clin. Invest. 111:291-297.
doi:10.1172/JCI200317748

18. Yuda, H., et al. 2004. Activated protein C inhibits bronchial hyperresponsiveness and Th2 cytokine expression in mice. Blood. 103:2196-2204.

19. Hays, S.R., and Fahy, J.V. 2003. The role of mucus in fatal asthma. Am. J. Med. 115:68-69.

20. Hoshino, M., Takahashi, M., and Aoike, N. 2001. Expression of vascular endothelial growth factor, basic fibroblast growth factor, and angiogenin immunoreactivity in asthmatic airways and its relationship to angiogenesis. J. Allergy Clin. Immunol. 107:295-301.

21. Babu, K.S., et al. 2003. Inhaled synthetic surfactant abolishes the early allergen-induced response in asthma. Eur. Respir. J. 21:1046-1049.

22. Cho, S.H., et al. 2001. Possible role of the $4 \mathrm{G} / 5 \mathrm{G}$ polymorphism of the plasminogen activator inhibitor 1 gene in the development of asthma. J. Allergy Clin. Immunol. 108:212-214.

23. Gabazza, E.C., et al. 1999. Thrombin in the airways of asthmatic patients. Lung. 177:253-262.

\section{Apoptotic mechanisms in Alzheimer neurofibrillary degeneration: cause or effect?}

Dennis W. Dickson

Departments of Pathology and Neuroscience, Mayo Clinic College of Medicine, Jacksonville, Florida, USA.

\begin{abstract}
Increasing evidence suggests that selective neuronal loss in neurodegenerative diseases involves activation of cysteine aspartyl proteases (caspases), which initiate and execute apoptosis. In Alzheimer disease both extracellular amyloid deposits and intracellular amyloid $\beta$ protein may activate caspases, leading to cleavage of nuclear and cytoskeletal proteins, including tau protein. Proteolysis of tau may be critical to neurofibrillary degeneration, which correlates with dementia (see the related article beginning on page 121).
\end{abstract}

Alzheimer disease (AD), the most common cause of dementia in the elderly, is associated with senile plaques and neurofibrillary tangles (NFTs), but the relationship between these two neuropathologic lesions has been difficult to discover. Senile plaques are heterogenous lesions composed of extracellular amyloid $\beta$ protein (A $\beta)$, dystrophic neuronal processes, and reactive glia (1), while NFTs are intracellular lesions composed of filamentous aggregates of the microtubuleassociated protein tau (2). Genetic factors have implicated $A \beta$ in the pathogenesis of $\mathrm{AD}$ since mutations are found in the $\mathrm{A} \beta$ precursor (APP) as well as in enzymes involved in the production of $\mathrm{A} \beta$ (reviewed

Nonstandard abbreviations used: Alzheimer disease $(\mathrm{AD})$; amyloid $\beta$ protein (A $\beta$ ); $\mathrm{A} \beta$ precursor (APP); neurofibrillary tangle (NFT); Parkinson disease (PD).

Conflict of interest: The author has declared that no conflict of interest exists.

Citation for this article: J. Clin. Invest. 114:23-27 (2004) doi:10.1172/JCI200422317. in ref. 3). Moreover, genes implicated in AD by linkage studies encode proteins that degrade $A \beta$, such as insulin-degrading enzyme. The major genetic risk factor for late-onset $\mathrm{AD}$, apolipoprotein $\mathrm{E}$, promotes $A \beta$ aggregation and colocalizes with $A \beta$ in senile plaques.

The conundrum that has plagued research on $\mathrm{AD}$ is that clinicopathologic studies have not shown strong correlations between cognitive impairment and $\mathrm{A} \beta$. In particular, the degree of cognitive impairment in AD is not as closely tied to the amount of amyloid deposited in the brain or the number of senile plaques as it is to the amount of abnormal tau protein in the brain and the density and distribution of NFTs $(4,5)$. This is not entirely surprising since NFTs are composed of proteins that are part of the neuronal cytoskeleton, which supports vital structural and dynamic neuronal functions. Amyloid, on the other hand, is a relatively innocuous extracellular deposit. It has been suggested that cytoskeletal disruption may act as the proximate cause of progressive synaptic and neuronal loss by interfering with axoplasmic and dendritic transport, which starves the cell of trophic support (6). This dysfunction and eventual death of neurons manifests clinically as cognitive impairment.

A major challenge of the amyloid cascade hypothesis for $\mathrm{AD}$ (Figure 1), which posits that amyloid formation leads to neuronal loss and dementia (7), is determining the link between $A \beta$, the protein most clearly linked to the cause of $\mathrm{AD}$, and tau, the protein that is most clearly associated with clinical manifestations of AD. The studies by Rissman and coworkers in this issue of the JCI (8) suggest that apoptotic mechanisms may be the missing link.

\section{Apoptosis in AD}

Apoptosis has been the focus of intense research in the last several decades as a means of controlling cell populations in normal development and inflammation through programmed cell death. Failure to control cell numbers through apoptosis is common in cancer, while excessive apoptosis is viewed to play a role in a number of neurologic disorders in addition to $\mathrm{AD}$, including stroke and Parkinson disease (PD) (9). 